\section{ARIEL \\ UNIVERSITY \\ PRESS}

Functional Differential Equations

Volume 27, No. 1-2, 2020, pp. 39-50

DOI: htps://doi.org/10.26351/FDE/27/1-2/5

ISSN: 0793-1786 (print), 2617-8605 (online)

\title{
NONLOCAL INTEGRAL BOUNDARY VALUE PROBLEMS WITH CAUSAL OPERATORS AND FRACTIONAL DERIVATIVES
}

\author{
G. WANG ${ }^{*}$, L. ZHANG ${ }^{\dagger}$ AND R. AGARWAL $\ddagger$
}

\begin{abstract}
This paper investigates the existence of solutions for a class of nonlocal integral boundary value problems with causal operators and fractional derivatives. By applying the monotone iterative technique and the method of lower and upper solutions, we provide sufficient conditions under which such problems have the maximal and minimal solutions or quasisolutions in a corresponding sector. Finally, an example illustrating how the theory can be applied in practice is also included.
\end{abstract}

Key Words. Causal operators; Fractional differential inequalities with positive linear operators; Nonlocal integral boundary conditions; Monotone iterative technique

AMS(MOS) subject classification. 30A10

1. Introduction. In recent years the study of differential equations with causal operators has attracted much attention. A causal operator is a non-anticipative operator. The term causal arises from the engineering and the notion of a causal operator turns out to be a powerful tool for unifying problems in ordinary differential equations, integro-differential equations, differential equations with finite or infinite delay, Volterra integral equations, and neutral functional equations, to name a few. A pioneering work has been reported by Corduneanu ([1],[2]), Drici et al. ([3]-[5]), Jankowski ([6],[7]) and Lupulescu ([8]). For some recent development on the topic, see ([9]-[12]) and the references therein.

Fractional differential equations arise in many engineering and scien-

* School of Mathematics and Computer Science, Shanxi Normal University, Linfen, Shanxi 041004, P. R. China

† School of Mathematics and Computer Science, Shanxi Normal University, Linfen, Shanxi 041004, P. R. China

$\ddagger$ Department of Mathematics, Texas A\&M University-Kingsville, Kingsville, TX 78363, USA 
tific disciplines as the mathematical modelling of systems and processes in the fields of physics, chemistry, aerodynamics, electrodynamics of complex medium, polymer rheology, etc. ([13]-[16]). The interest in the study of differential equations of fractional order lies in the fact that fractional derivatives provide an excellent tool for the description of memory and hereditary properties of various materials and processes. With this advantage, the fractional-order models are regarded as important mathematical tools for the better understanding of several real world problems in applied sciences. For some recent work on differential equations of fractional order, see ([17]-[30]) and the references therein.

Integral type of boundary conditions have recently been addressed by several researchers. The interest in the study of integral boundary conditions lies in the fact that it has various applications in applied fields such as blood flow problems, chemical engineering, thermoelasticity, underground water flow, population dynamics, and so forth. For a detailed description of the integral boundary conditions, we refer the reader to some recent papers ([31]-[38]) and the references therein. It has been observed that the limits of integration in the integral part of the boundary conditions are taken to be fixed, for instance, from 0 to $T$ in case the independent variable belongs to the interval $[0, T]$. It is imperative to note that the available literature on nonlocal boundary conditions is confined to the nonlocal parameters involvement in the solution or gradient of the solution of the problem. In our paper, a nonlocal type of integral boundary condition with limits of integration involving the parameters $0<\tau<T$ has been introduced. It is worth mentioning that, in practical situations, such nonlocal integral boundary conditions may be regarded as a continuous distribution of arbitrary finite length, for instance, see [39].

In this paper, we study the following nonlocal integral boundary value problems for fractional differential equation with causal operators

$$
\left\{\begin{aligned}
D^{\alpha} u(t) & =(Q u)(t), \\
\left.t^{1-\alpha} u(t)\right|_{t=0} & =t^{1-\alpha} \int_{0}^{\tau} w(s, u(s)) d s+x_{0},
\end{aligned}\right.
$$

where $t \in J=[0, T](T>0), E=C(J, \mathbb{R}), Q \in C(E, E)$ is a causal operator, $\tau \in(0, T], w \in C(J \times \mathbb{R}, \mathbb{R}), x_{0} \in \mathbb{R}$ and $D^{\alpha}$ is the RiemannLiouville fractional derivative of $u$ and $\alpha$ is such that $0<\alpha<1$.

To obtain approximate solutions of nonlinear differential equations, we also develop the monotone iterative technique. This technique, combined with the method of upper and lower solutions provides an effective mechanism to prove constructive existence results for nonlinear differential equations, the 
advantage and importance of the technique needs no special emphasis [40]. To the best of our knowledge, the problem of fractional differential equations with causal operators and nonlocal integral boundary conditions has not been attempted so far. It is also the first paper in which the monotone iterative method is applied to problems of the type (1).

The rest of the paper is organized as follows. In Section 2, we list several lemmas and a new comparison principle, which play an important role in the proof of main results. Further, to study the nonlinear problem (1), we consider the associated linear problem and obtain the uniqueness of the solutions to the associated linear problem. In Section 3, we formulate sufficient conditions which guarantee that problem (1) has maximal and minimal solutions. A one-sided Lipschitz condition (with corresponding linear operators) is imposed on the causal operator Q. In Section 4, by using the notion of coupled lower and upper solutions, we testify the existence of quasisolutions of the problem (1). In Section 5, an example is given to illustrate our main results.

\section{Preliminaries.}

For the convenience of the readers, in this section we first present some useful definitions and lemmas.

Definition 1. The Riemann-Liouville fractional derivative of order $\delta$ for a function $f(t)$ is defined by

$$
D^{\delta} f(t)=\frac{1}{\Gamma(n-\delta)}\left(\frac{d}{d t}\right)^{n} \int_{0}^{t}(t-s)^{n-\delta-1} f(s) d s, \quad n=[\delta]+1,
$$

provided the right hand side is defined pointwise on $(0, \infty)$.

DEFinition 2. The Riemann-Liouville fractional integral of order $\delta$ for a function $f$ is defined as

$$
I^{\delta} f(t)=\frac{1}{\Gamma(\delta)} \int_{0}^{t}(t-s)^{\delta-1} f(s) d s, \quad \delta>0
$$

provided that such integral exists.

Let $C_{1-\alpha}(J, \mathbb{R})=\left\{u \in C(0, T] ; t^{1-\alpha} u \in C(J, \mathbb{R})\right\}$.

Lemma 1. (Comparison result) Let $w \in C_{1-\alpha}(J, \mathbb{R})$ satisfies

$$
\left\{\begin{array}{l}
D^{\alpha} w(t) \leq-M(t) w(t)-(\mathcal{L} w)(t) \\
\left.t^{1-\alpha} w(t)\right|_{t=0} \leq 0
\end{array}\right.
$$

where $M(t)$ is bounded nonnegative integral function and $\mathcal{L} \in C(E, E)$ is a positive linear operator. Then, $w(t) \leq 0, \forall t \in(0, T]$. 
Proof. Suppose $w(t) \leq 0$ is not true, then there exist $t^{*}, t_{*} \in(0, T]$ such that

$$
w\left(t^{*}\right)=0, \quad w\left(t_{*}\right)>0
$$

and

$$
w(t) \leq 0, \quad t \in\left(0, t^{*}\right), \quad w(t)>0, \quad t \in\left(t^{*}, t_{*}\right) .
$$

Thus, if $t \in\left(t^{*}, t_{*}\right)$, we have $D^{\alpha} w(t) \leq 0$. Since

$$
D^{\alpha} w(t)=\frac{d}{d t} I^{1-\alpha} w(t)
$$

It follows that $I^{1-\alpha} w(t)$ is nonincreasing on $\left(t^{*}, t_{*}\right)$. Therefore, we get

$$
I^{1-\alpha} w(t)-I^{1-\alpha} w\left(t^{*}\right) \leq 0, \quad t \in\left(t^{*}, t_{*}\right) .
$$

On the other hand,

$$
\begin{aligned}
I^{1-\alpha} w(t)-I^{1-\alpha} w\left(t^{*}\right) & =\int_{0}^{t} \frac{(t-s)^{-\alpha}}{\Gamma(1-\alpha)} w(s) d s-\int_{0}^{t^{*}} \frac{\left(t^{*}-s\right)^{-\alpha}}{\Gamma(1-\alpha)} w(s) d s \\
& =\int_{0}^{t} \frac{(t-s)^{-\alpha}-\left(t^{*}-s\right)^{-\alpha}}{\Gamma(1-\alpha)} w(s) d s+\int_{t^{*}}^{t} \frac{(t-s)^{-\alpha}}{\Gamma(1-\alpha)} w(s) d s \\
& >0, t \in\left(t^{*}, t_{*}\right),
\end{aligned}
$$

which contradicts $(3)$. Thus, $w(t) \leq 0(t \in(0, T])$ holds. This completes the proof.

To study the nonlinear problem (1), we first consider the associated linear problem:

$$
\left\{\begin{aligned}
D^{\alpha} u(t) & =\sigma(t)-M(t) u(t)-(\mathcal{L} u)(t) \\
\left.t^{1-\alpha} u(t)\right|_{t=0} & =t^{1-\alpha} \int_{0}^{\tau} w(s, \eta(s)) d s+x_{0}
\end{aligned}\right.
$$

where $\sigma, \eta \in C(J, \mathbb{R})$ and $M(t), \mathcal{L}(t)$ are as in Lemma 1 . LEMMA 2. If

$$
I^{\alpha} M(t)+\frac{\|\mathcal{L}\| t^{\alpha}}{\Gamma(\alpha+1)}<1
$$

Then (5) has a unique solution $u \in C_{1-\alpha}(J, \mathbb{R})$. 
Proof. Obviously, $u(t) \in C_{1-\alpha}(J, \mathbb{R})$ is a solution of (5) if and only if $u(t) \in$ $C(J, R)$ is a solution of the following integral equation

$$
\begin{aligned}
u(t) & =\int_{0}^{t} \frac{(t-s)^{\alpha-1}}{\Gamma(\alpha)}[\sigma(s)-M(s) u(s)-(\mathcal{L} u)(s)] d s+\int_{0}^{\tau} w(s, \eta(s)) d s+x_{0} t^{\alpha-1} \\
& \triangleq T u(t) .
\end{aligned}
$$

For any $u, v \in C(J, \mathbb{R})$, by $(7)$ we have

$$
\begin{aligned}
|T u(t)-T v(t)| & \leq \int_{0}^{t} \frac{(t-s)^{\alpha-1}}{\Gamma(\alpha)}[M(s)|(u-v)(s)|+|(\mathcal{L} u)-(\mathcal{L} v)(s)|] d s \\
& \leq \int_{0}^{t} \frac{(t-s)^{\alpha-1}}{\Gamma(\alpha)}[M(s)+\|\mathcal{L}\|] d s\|u-v\|_{C} .
\end{aligned}
$$

Therefore, it follows that

$$
\|T u-T v\|_{C} \leq\left[I^{\alpha} M(t)+\frac{\|\mathcal{L}\| t^{\alpha}}{\Gamma(\alpha+1)}\right]\|u-v\|_{C}<\|u-v\|_{C} .
$$

Hence, $T$ is a contraction operator on $C(J, \mathbb{R})$. Consequently, it has a unique fixed point $u$, i.e. $u(t)$ is a unique solution of $(5)$.

\section{Maximal and minimal solutions of (1).}

In this section we shall use the monotone iterative technique to prove the existence of maximal and minimal solutions of nonlocal integral boundary value problem involving causal operators (1).

DeFinition 3. We say that $u \in C_{1-\alpha}(J, \mathbb{R})$ is a lower solution of $(1)$ if

$$
\left\{\begin{aligned}
D^{\alpha} u(t) & \leq(Q u)(t) \\
\left.t^{1-\alpha} u(t)\right|_{t=0} & \leq t^{1-\alpha} \int_{0}^{\tau} w(s, u(s)) d s+x_{0}
\end{aligned}\right.
$$

and it is an upper solution of (1), if the above inequalities are reversed.

THEOREM 1. Assume that

$\left(H_{1}\right) u_{0}, v_{0} \in C_{1-\alpha}(J, \mathbb{R})$ are the lower and upper solutions of $(1)$, respectively, and $u_{0}(t) \leq v_{0}(t)$.

$\left(\mathrm{H}_{2}\right)$ The following conditions

$$
\begin{gathered}
(Q u)(t)-(Q v)(t) \geq-M(t)(u-v)(t)-(\mathcal{L}(u-v))(t), \\
\int_{0}^{\tau}[w(s, u(s))-w(s, v(s))] d s \geq 0 .
\end{gathered}
$$


hold for $u_{0} \leq v \leq u<v_{0}$, here $M(t), \mathcal{L}(t)$ are as in Lemma 2 and satisfy (6). Then there exist monotone iterative sequences $\left\{u_{n}\right\},\left\{v_{n}\right\} \subset\left[u_{0}, v_{0}\right]$ which converge uniformly to the minimal and maximal solutions $u^{*}, v^{*}$ of (1) in $\left[u_{0}, v_{0}\right]$, respectively, where $\left\{u_{n}\right\},\left\{v_{n}\right\}$ are defined by

$$
\begin{aligned}
u_{n}(t)= & \int_{0}^{t} \frac{(t-s)^{\alpha-1}}{\Gamma(\alpha)}\left[\left(Q u_{n-1}\right)(s)-M(s)\left(u_{n}-u_{n-1}\right)(s)-\left(\mathcal{L}\left(u_{n}-u_{n-1}\right)\right)(s)\right] d s \\
& +\int_{0}^{\tau} w\left(s, u_{n-1}(s)\right) d s+x_{0} t^{\alpha-1},
\end{aligned}
$$

$$
\begin{aligned}
v_{n}(t)= & \int_{0}^{t} \frac{(t-s)^{\alpha-1}}{\Gamma(\alpha)}\left[\left(Q v_{n-1}\right)(s)-M(s)\left(v_{n}-v_{n-1}\right)(s)-\left(\mathcal{L}\left(v_{n}-v_{n-1}\right)\right)(s)\right] d s \\
& +\int_{0}^{\tau} w\left(s, v_{n-1}(s)\right) d s+x_{0} t^{\alpha-1}
\end{aligned}
$$

and

$$
u_{0} \leq u_{1} \leq \cdots \leq u_{n} \leq \cdots \leq u^{*} \leq v^{*} \leq \cdots \leq v_{n} \leq \cdots \leq v_{1} \leq v_{0}
$$

Proof. For any $u_{n-1}, v_{n-1} \in C(J, \mathbb{R})$, by Lemma 2, we know that (9) and (10) have unique solutions $u_{n}, v_{n} \in C(J, \mathbb{R})$, respectively. To complete the proof we need the following three steps:

Step 1: $u_{n-1} \leq u_{n}$ and $v_{n} \leq v_{n-1}, n=1,2, \cdots$.

$$
\left\{\begin{aligned}
D^{\alpha} u_{n}(t)+M(t) u_{n}(t)+\left(\mathcal{L} u_{n}\right)(t) & =\left(Q u_{n-1}\right)(t)+M(t) u_{n-1}(t)+\left(\mathcal{L} u_{n-1}\right)(t) \\
\left.t^{1-\alpha} u_{n}(t)\right|_{t=0} & =t^{1-\alpha} \int_{0}^{\tau} w\left(s, u_{n-1}(s)\right) d s+x_{0}
\end{aligned}\right.
$$

and

$$
\left\{\begin{aligned}
D^{\alpha} v_{n}(t)+M(t) v_{n}(t)+\left(\mathcal{L} v_{n}\right)(t) & =\left(Q v_{n-1}\right)(t)+M(t) v_{n-1}(t)+\left(\mathcal{L} v_{n-1}\right)(t) \\
\left.t^{1-\alpha} v_{n}(t)\right|_{t=0} & =t^{1-\alpha} \int_{0}^{\tau} w\left(s, v_{n-1}(s)\right) d s+x_{0}
\end{aligned}\right.
$$

Thus, $u_{1}$ satisfies

$$
\left\{\begin{aligned}
D^{\alpha} u_{1}(t)+M(t) u_{1}(t)+\left(\mathcal{L} u_{1}\right)(t) & =\left(Q u_{0}\right)(t)+M(t) u_{0}(t)+\left(\mathcal{L} u_{0}\right)(t), \\
\left.t^{1-\alpha} u_{1}(t)\right|_{t=0} & =t^{1-\alpha} \int_{0}^{\tau} w\left(s, u_{0}(s)\right) d s+x_{0}
\end{aligned}\right.
$$


Let $p(t)=u_{0}(t)-u_{1}(t)$, then by condition $\left(H_{1}\right)$, we have

$$
D^{\alpha} p(t)+M(t) p(t)+(\mathcal{L} p)(t)=D^{\alpha} u_{0}(t)-\left(Q u_{0}\right)(t) \leq 0 .
$$

In addition,

$$
\left.t^{1-\alpha} p(t)\right|_{t=0}=\left.t^{1-\alpha} u_{0}(t)\right|_{t=0}-\left.t^{1-\alpha} u_{1}(t)\right|_{t=0} \leq 0 .
$$

Hence, by Lemma $1, p(t) \leq 0$, which implies that $u_{0}(t) \leq u_{1}(t)$.

Now by mathematical induction it easily follows that

$$
u_{0} \leq u_{1} \leq \cdots \leq u_{n} \leq \cdots, n=1,2, \cdots
$$

Similarly, we have

$$
\cdots \leq v_{n} \leq \cdots \leq v_{1} \leq v_{0}, n=1,2, \cdots .
$$

Step 2: $u_{n} \leq v_{n}, n=1,2, \cdots$.

Let $p(t)=u_{1}(t)-v_{1}(t)$, then by condition $\left(H_{2}\right)$, we have

$D^{\alpha} p(t)+M(t) p(t)+(\mathcal{L} p)(t)=\left(Q u_{0}\right)(t)+M(t) u_{0}(t)+\left(\mathcal{L} u_{0}\right)(t)-\left(Q v_{0}\right)(t)-M(t) v_{0}(t)-\left(\mathcal{L} v_{0}\right)(t) \leq 0$.

Moreover,

$\left.t^{1-\alpha} p(t)\right|_{t=0}=\left.t^{1-\alpha} u_{1}(t)\right|_{t=0}-\left.t^{1-\alpha} v_{1}(t)\right|_{t=0}=t^{1-\alpha} \int_{0}^{\tau}\left[w\left(s, u_{0}(s)\right)-w\left(s, v_{0}(s)\right)\right] d s \leq 0$.

Thus by Lemma 1 , we have $p(t) \leq 0$, which implies that $u_{1}(t) \leq v_{1}(t)$. Now mathematical induction leads to $u_{n} \leq v_{n}, n=1,2, \cdots$.

Combining Steps 1 and 2, we find

$$
u_{0} \leq u_{1} \leq \cdots \leq u_{n} \leq \cdots \leq v_{n} \leq \cdots \leq v_{1} \leq v_{0} .
$$

Thus, using standard arguments, we find that $\lim _{n \rightarrow \infty} u_{n}(t)=u^{*}(t)$ and $\lim _{n \rightarrow \infty} v_{n}(t)=$ $v^{*}(t)$ uniformly on $J$, and $u^{*}(t), v^{*}(t)$ are solutions of (1). Hence, (11) holds.

Step 3: $u^{*}(t), v^{*}(t)$ are the minimal and maximal solutions of (1).

Assume that $\omega(t) \in\left[u_{0}(t), v_{0}(t)\right]$ be any solution of $(1)$, i.e.,

$$
\left\{\begin{aligned}
D^{\alpha} \omega(t) & =(Q \omega)(t) \\
\left.t^{1-\alpha} \omega(t)\right|_{t=0} & =t^{1-\alpha} \int_{0}^{\tau} w(s, \omega(s)) d s+x_{0} .
\end{aligned}\right.
$$

Combining $(12),(13)$ and $\left(H_{2}\right)$, and using Lemma 1 , it follows by induction that

$$
u_{n}(t) \leq \omega(t) \leq v_{n}(t), \quad n=1,2, \cdots .
$$

Now, letting $n \rightarrow+\infty$ in (18), we find $u^{*} \leq \omega \leq v^{*}$. That is, $u^{*}, v^{*}$ are the minimal and maximal solutions of $(1)$ in $\left[u_{0}, v_{0}\right]$. 


\section{Quasisolutions of (1).}

This section deals with the existence of quasisolutions for nonlocal integral boundary value problem (1).

Definition 4. We say that $u, v \in C_{1-\alpha}(J, \mathbb{R})$ are coupled lower and upper solutions of $(1)$, if

$$
\left\{\begin{aligned}
D^{\alpha} u(t) & \leq(Q u)(t) \\
\left.t^{1-\alpha} u(t)\right|_{t=0} & \leq t^{1-\alpha} \int_{0}^{\tau} w(s, v(s)) d s+x_{0} \\
D^{\alpha} v(t) & \geq(Q v)(t) \\
\left.t^{1-\alpha} v(t)\right|_{t=0} & \geq t^{1-\alpha} \int_{0}^{\tau} w(s, u(s)) d s+x_{0},
\end{aligned}\right.
$$

Definition 5. We say that $U, V \in C_{1-\alpha}(J, \mathbb{R})$ are quasisolutions of $(1)$, if

$$
\left\{\begin{aligned}
D^{\alpha} U(t) & =(Q U)(t) \\
\left.t^{1-\alpha} U(t)\right|_{t=0} & =t^{1-\alpha} \int_{0}^{\tau} w(s, V(s)) d s+x_{0} \\
D^{\alpha} V(t) & =(Q V)(t) \\
\left.t^{1-\alpha} V(t)\right|_{t=0} & =t^{1-\alpha} \int_{0}^{\tau} w(s, U(s)) d s+x_{0}
\end{aligned}\right.
$$

TheOREM 2. Assume that

$\left(H_{1}^{\prime}\right) u_{0}, v_{0} \in C_{1-\alpha}(J, \mathbb{R})$ are the coupled lower and upper solutions of (1), respectively, and $u_{0}(t) \leq v_{0}(t)$.

$\left(H_{2}^{\prime}\right)$ The following conditions

$$
\begin{gathered}
(Q u)(t)-(Q v)(t) \geq-M(t)(u-v)(t)-(\mathcal{L}(u-v))(t), \\
\int_{0}^{\tau}[w(s, u(s))-w(s, v(s))] d s \leq 0 .
\end{gathered}
$$

hold for $u_{0} \leq v \leq u<v_{0}$, here $M(t), \mathcal{L}(t)$ are as in Lemma 2 and satisfy (6).

Then there exist monotone iterative sequences $\left\{u_{n}\right\},\left\{v_{n}\right\} \subset\left[u_{0}, v_{0}\right]$ which converge uniformly to the quasisolutions $u^{*}, v^{*}$ of (1) in $\left[u_{0}, v_{0}\right]$, respectively, where $\left\{u_{n}\right\},\left\{v_{n}\right\}$ are defined as

$$
\begin{aligned}
u_{n}(t)= & \int_{0}^{t} \frac{(t-s)^{\alpha-1}}{\Gamma(\alpha)}\left[\left(Q u_{n-1}\right)(s)-M(s)\left(u_{n}-u_{n-1}\right)(s)-\left(\mathcal{L}\left(u_{n}-u_{n-1}\right)\right)(s)\right] d s \\
& +\int_{0}^{\tau} w\left(s, v_{n-1}(s)\right) d s+x_{0} t^{\alpha-1}
\end{aligned}
$$




$$
\begin{aligned}
v_{n}(t)= & \int_{0}^{t} \frac{(t-s)^{\alpha-1}}{\Gamma(\alpha)}\left[\left(Q v_{n-1}\right)(s)-M(s)\left(v_{n}-v_{n-1}\right)(s)-\left(\mathcal{L}\left(v_{n}-v_{n-1}\right)\right)(s)\right] d s \\
& +\int_{0}^{\tau} w\left(s, u_{n-1}(s)\right) d s+x_{0} t^{\alpha-1},
\end{aligned}
$$

and

$$
u_{0} \leq u_{1} \leq \cdots \leq u_{n} \leq \cdots \leq u^{*} \leq v^{*} \leq \cdots \leq v_{n} \leq \cdots \leq v_{1} \leq v_{0} .
$$

Proof. The proof is similar to that of Theorem 1.

5. Example. Consider the problem:

$$
\left\{\begin{array}{c}
D^{\alpha} u(t)=-M(t) u(t)+M(t) \sin \left(\frac{\pi}{2} u\left(\frac{t^{2}}{3}\right)\right)-3 \int_{0}^{t} t^{2} s^{3} u(s) d s, \\
\left.t^{1-\alpha} u(t)\right|_{t=0}=t^{1-\alpha} \int_{0}^{\frac{2}{3}}\left(s^{2}+\frac{u^{3}(s)}{27}\right) d s
\end{array}\right.
$$

here $t \in J=[0,1], D^{\alpha}$ is the standard Riemann-Liouville fractional derivative of order $0<\alpha<1$ and $M(t)$ is bounded nonnegative integrable function.

Clearly,

$$
\left\{\begin{array}{l}
(Q u)(t)=-M(t) u(t)+M(t) \sin \left(\frac{\pi}{2} u\left(\frac{t^{2}}{3}\right)\right)-3 \int_{0}^{t} t^{2} s^{3} u(s) d s \\
w(t, u(t))=t^{2}+\frac{u^{3}(t)}{27}
\end{array}\right.
$$

Take $u_{0}(t)=0, v_{0}(t)=1$. It is not difficult to verify that $u_{0}, v_{0}$ are lower and upper solutions of $(22)$, respectively, and $u_{0} \leq v_{0}$. Thus, condition $\left(H_{1}\right)$ holds. In addition, we have

$$
\begin{gathered}
(Q u)(t)-(Q v)(t) \geq-M(t)(u-v)(t)-(\mathcal{L}(u-v))(t), \\
\int_{0}^{\tau}[w(s, u(s))-w(s, v(s))] d s \geq 0,
\end{gathered}
$$

for $u_{0} \leq v \leq u<v_{0}$, where $(\mathcal{L} u)(t)=3 \int_{0}^{t} t^{2} s^{3} u(s) d s$.

If we assume that

$$
I^{\alpha} M(t)+\frac{\|\mathcal{L}\| t^{\alpha}}{\Gamma(\alpha+1)}<1
$$

Then all conditions of Theorem 1 are satisfied. Therefore, by Theorem 1 , there exist monotone iterative sequences $\left\{u_{n}\right\},\left\{v_{n}\right\} \subset\left[u_{0}, v_{0}\right]$ which converge uniformly to the minimal and maximal solutions $u^{*}, v^{*}$ of $(1)$ in $\left[u_{0}, v_{0}\right]$, respectively. 


\section{REFERENCES}

[1] C. Corduneanu, Functional Equations with Causal Operators, Stability and Control: Theory, Methods and Applications, Taylor Francis, London, 16 (2002).,

[2] C. Corduneanu, Existence of solutions for neutral functional differential equations with causal operators, J. Differential Equations, 168 (2000), 93-101

[3] Z. Drici, F. McRae, J. Vasundhara Devi, Set differential equations with causal operators, Math. Probl. Eng.,2 (2005), 185-194.

[4] Z. Drici, F. McRae, J. Vasundhara Devi, Differential equations with causal operators in a Banach space, Nonlinear Anal.,62, (2005), 301-313.

[5] Z. Drici, F.A. McRae, J. Vasundhara Devi, Monotone iterative technique for periodic value problems with causal operators, Nonlinear Anal., 64 (2006), 1271-1277.

[6] T. Jankowski, Nonlinear boundary value problems for second order differential equations with causal operators, J. Math. Anal. Appl., 332 (2007), 1380-1392.

[7] T. Jankowski, Boundary value problems with causal operators, Nonlinear Anal., 68 (2008), 3625-3632.

[8] V. Lupulescu, Causal functional differential equations in Banach spaces, Nonlinear Anal., 69 (2008), 4787-4795.

[9] V. Lakshmikantham, S. Leela, Z. Drici, F.A. McRae, Theory of Causal Differential Equations, Atlantis Studies in Mathematics for Engineering and Science World Scientific, 5 (2010).

[10] J. Vasundhara Devi, Existence, uniqueness of solutions for set differential equations involving causal operators with memory, Eur. J. Pure Appl. Math., 3 (2010), 737-747.

[11] V. Obukhovskii, P. Zecca, On certain classes of functional inclusions with causal operators in Banach spaces, Nonlinear Anal., 74 (2011) 2765-2777.

[12] R. Agarwal, Y. Zhou, J. Wang, X. Luo, Fractional functional differential equations with causal operators in Banach spaces, Mathematical and Computer Modelling (2011) doi:10.1016/j.mcm.2011.04.016.

[13] S. Samko, A. Kilbas, O. Marichev, Fractional Integrals and Derivatives, Theory and Applications, Gordon and Breach, London, 1993.

[14] I. Podlubny, Fractional Differential Equations, Academic Press, San Diego, 1999.

[15] A. Kilbas, H. Srivastava, J. Trujillo, Theory and Applications of Fractional Differential Equations, North-Holland Mathematics Studies, Elsevier Science B.V., Amsterdam, 204 (2006).

[16] J. Sabatier, O. Agrawal, J. Machado (Eds.), Advances in Fractional Calculus: Theoretical Developments and Applications in Physics and Engineering, Springer, Dordrecht, 2007.

[17] R. Agarwal, B. Andrade, C. Cuevas, Weighted pseudo-almost periodic solutions of a class of semilinear fractional differential equations, Nonlinear Anal. Real World Appl., 11 (2010), 3532-3554.

[18] R. Agarwal, V. Lakshmikantham, J. Nieto, On the concept of solution for fractional differential equations with uncertainty, Nonlinear Anal., 72 (2010), 2859-2862.

[19] R.P. Agarwal, Y. Zhou, Y. He, Existence of fractional neutral functional differential equations, Comput. Math. Appl., 59 (2010), 1095-1100.

[20] R. P. Agarwal, B. Ahmad, Existence of solutions for impulsive anti-periodic boundary value problems of fractional semilinear evolution equations, Dyn. Contin. Discrete Impuls. Syst., Ser. A, Math. Anal., to appear.

[21] M. Al-Refai, M. Ali Hajji, Monotone iterative sequences for nonlinear boundary value 
problems of fractional order, Nonlinear Anal., 74 (2011) 3531-3539.

[22] Y. Zhou, F. Jiao, J. Li, Existence and uniqueness for p-type fractional neutral differential equations, Nonlinear Anal. 71 (2009) 2724-2733.

[23] Y. Zhou, F. Jiao, Nonlocal Cauchy problem for fractional evolution equations, Nonlinear Anal., RWA 11 (2010) 4465-4475.

[24] J. Wang, Y. Zhou, A class of fractional evolution equations and optimal controls, Nonlinear Anal. 12 (2011) 262-272.

[25] G. Wang, B. Ahmad, L. Zhang, Impulsive anti-periodic boundary value problem for nonlinear differential equations of fractional order, Nonlinear Anal., 74 (2011) 792-804.

[26] G. Wang, B. Ahmad, L. Zhang, Some existence results for impulsive nonlinear fractional differential equations with mixed boundary conditions, Comput. Math. Appl., 62 (2011), 1389-1397.

[27] G. Wang, S. K. Ntouyas, L. Zhang, Positive solutions of the three-point boundary value problem for fractional-order differential equations with an advanced argument, Adv. Differ. Equ-NY, 22011.

[28] B. Ahmad, G. Wang, A study of an impulsive four-point nonlocal boundary value problem of nonlinear fractional differential equations, Comput. Math. Appl., 62 (2011), 1341-1349.

[29] B. Ahmad, S. Sivasundaram, On four-point nonlocal boundary value problems of nonlinear integro-differential equations of fractional order, Appl. Math. Comput. 217 (2010), 480-487.

[30] B. Ahmad, J.J. Nieto, Existence of solutions for nonlocal boundary value problems of higher order nonlinear fractional differential equations, Abstr. Appl. Anal., 2009 (2009), Article ID 494720, 9 pages.

[31] B. Ahmad, A. Alsaedi, B. Alghamdi, Analytic approximation ofsolutions of the forced Duffing equation with integral boundary conditions, Nonlinear Anal., RWA 9 (2008,) 1727-1740.

[32] B. Ahmad, S.K. Ntouyas, A. Alsaedi, New existence results for nonlinear fractional differential equations with three-point integral boundary conditions, Adv. Differ. Equ-NY, 2011, Article ID 107384, 11 pages.

[33] J.Q. Jiang, L.S. Liu Y.H. Wu, Second-order nonlinear singular Sturm-Liouville problems with integral boundary conditions, Appl. Math. Comput., 215 (2009), 15731582.

[34] X.M. Zhang, M.Q. Feng, W.G. Ge, Existence result of second-order differential equations with integral boundary conditions at resonance, J. Math. Anal. Appl., 353 (2009), 311-319.

[35] T. Jankowski, Positive solutions for fourth-order differential equations with deviating arguments and integral boundary conditions, Nonlinear Anal., 73 (2010), 12891299.

[36] G. Wang, Boundary value problems for systems of nonlinear integro-differential equations with deviating arguments, J. Comput. Appl. Math., 234 (2010), 1356-1363.

[37] G. Wang, G. Song, L. Zhang, Integral boundary value problems for first order integrodifferential equations with deviating arguments, J. Comput. Appl. Math., 225 (2009), 602-611.

[38] G. Wang, L. Zhang, G. Song, Integral boundary value problem for impulsive integro-differential equations in Banach spaces, $J$ Appl Math Comput., DOI 10.1007/s12190-010-0450-3.

[39] S. Asghar, B. Ahmad, M. Ayub, Diffraction from an absorbing half plane due to a 
G. WANG, L. ZHANG, R. AGARWAL

finite cylindrical source, Acustica-Acta Acustica, 82 (1996), 365-367.

[40] G. Ladde, V. Lakshmikantham, A.Vatsala, Monotone Iterative Techniques for Nonlinear Differential Equations, Pitman Pub. Co., Boston, 1985. 